\title{
CARACTERIZACIÓN DE CRISTALES LÍQUIDOS POR MICROSCOPÍA ÓPTICA EN SISTEMAS SURFACTANTE POLIETOXILADO-ALCANO-AGUA
}

\author{
Gerson Chávez*, Irán Parra, Milangel Luzardo, Bélgica Bravo y Nelson Márquez \\ Laboratorio de Petroquímica y Surfactantes, Universidad del Zulia, Maracaibo, Zulia, Venezuela
}

Recebido em 5/3/13; aceito em 27/6/13; publicado na web em 2/8/13

\begin{abstract}
CHARACTERIZATION OF LIQUID CRYSTALS BY OPTICAL MICROSCOPY IN POLYETHOXYLATED SURFACTANTALKANE-WATER SYSTEMS. The phase behavior of an alcohol polyethoxylated surfactant with decane and dodecane oil phase varying the water proportion from 5 to $90 \%$ to determine compositions in which the formation of liquid crystals and microemulsions ocurred was investigated. Pseudoternary phase diagrams were built to represent the regions of liquid crystals, biphases and microemulsions. Polarized light optical microscopy was used for the analysis and characterization of the separate phases. The micrographs obtained showed characteristics of hexagonal and lamellar phases of liquid crystal, isotropic phases, microemulsions and vesicles. This study is important to propose hypothesis regarding the factors determining the formation and stability of phases composed by surfactant/oil/water systems.
\end{abstract}

Keywords: optical microscopy; liquid crystal; alcohol polyethoxylated.

\section{INTRODUCCIÓN}

Los surfactantes o tensoactivos en sistemas agua/aceite forman una gran variedad de estructuras de autoasociación entre las cuales se pueden mencionar las microemulsiones y los cristales líquidos. ${ }^{1}$ Una microemulsión es un fluido isotrópico, de baja viscosidad y termodinámicamente estable en la cual cantidades proporcionadas de dos fases inmiscibles (como agua y aceite) están dispersas en una sola fase por adición de una cantidad apropiada de un surfactante. Las microemulsiones están formadas por gotas con diámetros mayores a $100 \mathrm{~nm}$, y tanto las tipo agua en aceite (w/o) como aceite en agua $(\mathrm{o} / \mathrm{w})$ se pueden obtener con un mismo surfactante, pero variando la composición de las fases inmiscibles, el ambiente fisicoquímico o la temperatura. ${ }^{2}$ Las microemulsiones han sido extensamente estudiadas, y se han reportado una variedad de investigaciones relacionadas con su composición, propiedades y estructuras. ${ }^{3}$ Algunos autores indican que esta fase puede ser obtenida a partir de otra con propiedades particulares, como los cristales líquidos. ${ }^{4,5}$ Los cristales líquidos, conocidos también como mesofases, fases intermedias o semisólidos, poseen una estructura con un grado de orden intermedio a nivel molecular entre los líquidos y los sólidos cristalinos. A diferencia de los líquidos ordinarios, en los cristales líquidos las moléculas o iones que los forman se disponen de manera relativamente ordenada, dando origen a un estado mesomórfico que poseen un orden de largo alcance en lo que respecta a la orientación molecular y un orden parcial, o bien desorden, en lo referente a la posición de las moléculas. ${ }^{6}$ En el caso de los surfactantes, las moléculas individuales dentro de la mesofase poseen una orientación restringida y una libertad relativa de movimiento, y pueden originar cristales líquidos termotrópicos y liotrópicos. Los primeros dependen del efecto de la temperatura sobre la formación del tipo de mesofase, mientras que los cristales líquidos liotrópicos se presentan en un cierto intervalo de temperatura y concentración cuando los surfactantes se dispersan en un líquido. ${ }^{7} \mathrm{Si}$ la concentración del surfactante en agua es baja el cristal líquido se encuentra disuelto, pero si se alcanza o sobrepasa la concentración micelar crítica entonces se forman asociaciones micelares. Por encima de una cierta concentración, que depende

*e-mail: gchavez@fec.luz.edu.ve de la naturaleza del surfactante, del sistema y la temperatura, las micelas se agrupan en una estructura ordenada y forman un cristal líquido liotrópico. Los cristales líquidos más comunes encontrados en los sistemas surfactante/agua/aceite son de estructura laminar y hexagonal (Figura 1). La fase de cristal líquido laminar está formada por bicapas de moléculas tensoactivas que se alternan con capas de agua (Figura 1A). Es una mesofase ligeramente translúcida que exhibe propiedades semifluidas. Por otro lado, la estructura de fase de cristal líquido hexagonal consiste en una disposición ordenada de micelas cilíndricas (Figura 1B). Desde el punto de vista macroscópico, esta mesofase se asemeja a un gel transparente exhibiendo una viscosidad mayor que la del cristal líquido laminar. ${ }^{5}$

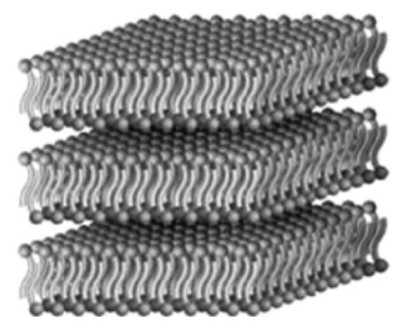

(A)

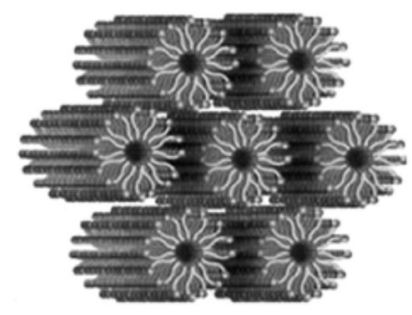

(B)
Figura 1. Representación del ordenamiento estructural en cristales líquidos de tipo: (A) laminar (con bicapas de surfactantes), (B) hexagonal (con micelas cilíndricas de surfactantes)

Las fases de cristal líquido presentan el fenómeno de la birrefringencia o doble refracción: un rayo de luz incidente se divide en otros dos que están polarizados perpendicularmente entre sí. El fenómeno de la doble refracción es responsable de la imagen en un microscopio con luz polarizada, a la que se denomina textura óptica, que se observa entre polarizadores cruzados. ${ }^{8}$

La posibilidad de disponer de formulaciones con cristales líquidos de transparencia óptica y apariencia gelificada, es altamente apreciada en diferentes campos de aplicación tales como cosméticos, farmacéuticas y alimentarias. ${ }^{9,10}$ En el presente trabajo se describe un estudio del comportamiento de fase de un alcohol polietoxilado, como surfactante no iónico, con dos alcanos y agua para la obtención 
de fases con propiedades de cristales líquidos liotrópicos y microemulsiones, y su caracterización por microscopía óptica.

\section{PARTE EXPERIMENTAL}

Se utilizó un surfactante no iónico de tipo alcohol polietoxilado (dodecil éter tetraetoxilado) conocido como Brij 30 (Aldrich, 98\%). Como aceites o fase oleica se utilizó el decano y dodecano (Merck, 99\%), y agua destilada y deionizada. Para el comportamiento de fases, a una mezcla surfactante/aceite se añadió lentamente el agua manteniendo una agitación constante (Vortex a 1000 rpm). En cada adición se observó el tipo de fase obtenida y se calculó la composición en masa de la mezcla surfactante/agua/aceite. Se empleó un microscopio óptico marca Leica modelo CTR6000 para caracterizar las fases de cristal líquido y observar la aparición de microemulsiones. Todas las micrografías fueron captadas a una amplitud de $20 \mu \mathrm{m}$. Se utilizó la luz polarizada (radiación visible entre polarizadores cruzados) para identificar la presencia y tipo de cristal líquido a través de la birrefringencia de las fases. La luz normal se empleó para observar los microemulsiones.

\section{RESULTADOS Y DISCUSIÓN}

En este trabajo se estudió la formación de cristales líquidos liotrópicos y microemulsiones en sistemas conteniendo un surfactante no iónico tipo alcohol polietoxilado, agua y una fase oleica (decano y dodecano), a través de los diagramas pseudoternarios de los tres componentes. La notación de las fases se realizó según el comportamiento frente a polarizadores cruzados y en un microscopio óptico con luz polarizada. Los límites de las regiones se trazaron desde los puntos experimentales obtenidos de la observación directa. A continuación se describe el comportamiento de fases del sistema surfactante/agua/ decano y surfactante/agua/dodecano, utilizados como modelos para la obtención de las fases de interés.

En la Figura 2 se presenta el diagrama pseudoternario del sistema surfactante/agua/decano a $25^{\circ} \mathrm{C}$. Se distinguen varias regiones: 1) w/o: región líquida isotrópica que se extiende a lo largo del eje decano-surfactante conformada por una microemulsión de agua-en-aceite. Esta fase se observó hasta un contenido máximo de 14\% de agua correspondiente a una relación decano/surfactante igual a 48/38. La estructura y composición de esta región corresponde a micelas inversas; ${ }^{8-11}$ 2) L $\alpha$ : región con estructura de cristal líquido, que se encuentra en la línea de composición surfactante/agua entre 16 a 56 $\%$ de agua, y hacia el vértice del decano hasta un $62 \%$, presentando cristales líquidos hexagonales y laminares (Figura 3); 3) ML $\alpha$ : región multifásica conformada por microemulsiones w/o y o/w en equilibrio con cristales líquidos que se caracterizan por su birrefringencia al movimiento. Esta región se encontró entre un 56 a $96 \%$ de agua a lo largo de la línea de composición agua/surfactante, y a partir de un contenido de decano de $4 \%$ hasta $59 \%$. Esta región se comportó como un sistema con una composición de cristal líquido donde no se observó la separación de las fases al equilibrio; 4) o/w: región líquida isotrópica que se extiende a lo largo del eje decano/agua conformada por una microemulsión tipo aceite-en-agua. Esta fase se observó hasta un contenido máximo de $9 \%$ del surfactante, correspondiente a una relación decano/agua igual a 50/41. La estructura de esta región pertenece a la fase cúbica bicontinua de tipo esponja. ${ }^{8-11}$ La zona de composición de esta región corresponde a la inmiscibilidad de dos fases líquidas, que de acuerdo a la separación de las fases pertenece a un sistema bifásico tipo Winsor I formado por una fase acuosa turbia (microemulsión o/w) y por una fase de decano en exceso; 5) Fw+L $\alpha$ : región de fase bicontinua formada por una microemulsión o/w separada de una fase en exceso de decano que coexiste en equilibrio con una fase de cristal líquido; 6) Fo+L $\alpha$ : región de fase bicontinua formada por una microemulsión w/o separada de una fase en exceso de agua que coexiste en equilibrio con una fase de cristal líquido; 7) V: región de dispersión de vesículas o liposomas en medio acuoso que se separan en una fase acuosa y una de cristales líquidos. Se extiende desde el vértice acuoso entre 56 a $97 \%$ de agua hasta un $44 \%$ del surfactante y conteniendo una concentración máxima de decano de $4,3 \%$. Esta fase fue definida por microscopía óptica ya que presentó birrefringencia con la formación de cruces de extinción de estructuras uniáxicas negativas (Figura 4c). Esta dispersión de vesículas es estable y al alcanzar el equilibrio se separa en dos fases, una fase acuosa (w) en equilibrio con una fase de cristal líquido $(L \alpha)$. También se forman vesículas en medio oleico a concentraciones de surfactante entre 7 y $20 \%$ que al alcanzar el equilibrio se separan en dos fases, una fase oleica y otra fase de cristal líquido, pero el límite de esta región no se pudo determinar.

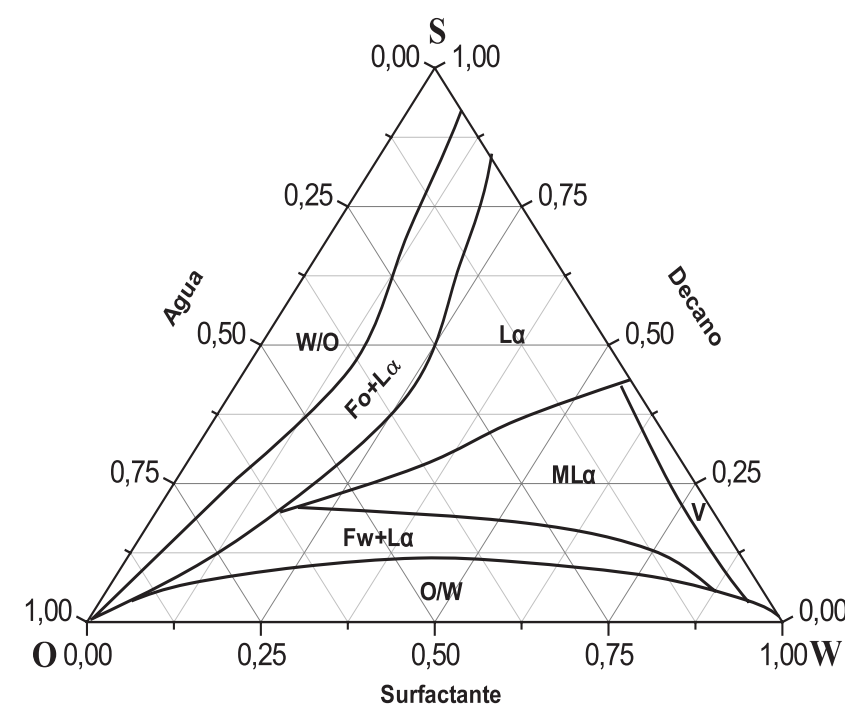

Figura 2. Diagrama ternario para el comportamiento de fases del sistema surfactantelagua/decano, a $25^{\circ} \mathrm{C}$

Las fases del diagrama pseudoternario de la Figura 2 fueron caracterizadas por microscopía óptica con luz polarizada, y las micrografías correspondientes se tomaron con una ampliación de $20 \mu \mathrm{m}$. En la Figura 3 se muestran las micrografías de las fases de cristales líquidos. A baja composición de decano, entre 44 a $84 \%$ de surfactante se encontraron cristales líquidos de tipo laminar nemáticos (Figura 3a), que se caracterizan por presentar una textura de tipo Grandjean con presencia de cruces de extinción de estructuras uniáxicas positivas. ${ }^{12}$ Esta textura es característica de cristales líquidos laminares y las cruces de extinción se muestran en la micrografía ampliada a 100x (Figura 3b). Los cristales líquidos laminares están constituidos por moléculas de surfactante en forma de bicapas planas separadas por una fase acuosa, como se muestra en la Figura 1a. A mayor concentración de decano aparecieron cristales líquidos hexagonales esmécticos (Figura 3c), los cuales se caracterizan por presentar una estructura de tipo abanico cónica focal dando origen a relieves. Este tipo de cristal líquido se origina a alta concentración de moléculas de surfactante en la cual forman barras cilíndricas largas a lo largo de un eje con arreglo hexagonal (Figura 1b). ${ }^{13}$

En la Figura 4 se muestran las micrografías de las fases de microemulsión y vesículas. Dependiendo de la composición y la cantidad de micelas formadas, estas fases pueden ser isotrópicas. Este fue el caso para la microemulsión w/o donde fue necesario tomar la fotografía con luz normal a un sistema agua/surfactante/decano con composición 4:70:26. Es notable la formación de gotas de la fase dispersa (agua), 

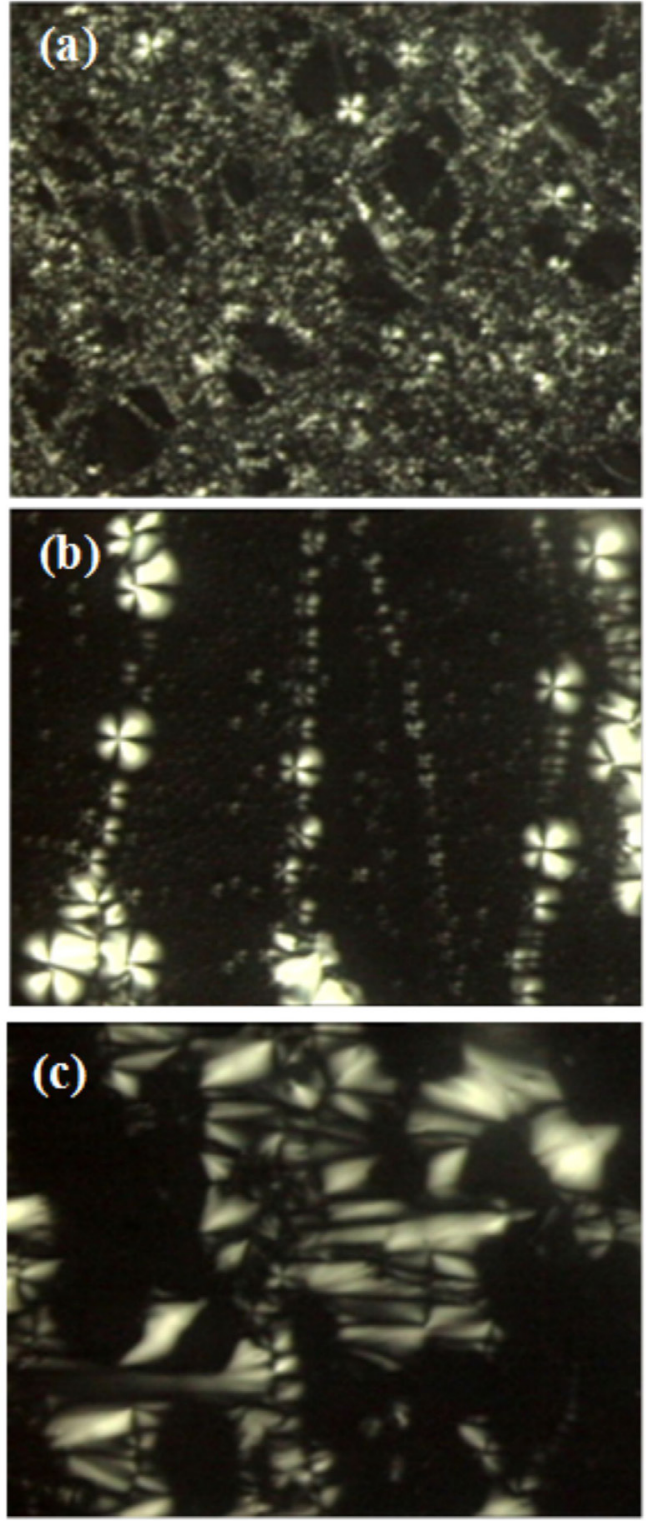

Figura 3. Micrografías (escala $20 \mu \mathrm{m}$ ) de la fase de cristales líquidos: (a) tipo laminar, con su respectiva ampliación a $100 x(b)$, y de tipo hexagonal (c), para el sistema surfactante/agua/decano

lo cual también se observa en la micrografía de la microemulsión o/w (Figura 4b) tomada para una composición agua/surfactante/decano 75:6:19, donde la fase dispersa es la oleica.

La fase de vesículas o liposomas se caracteriza por presentar birrefringencia a la luz con la formación de cruces de extinción, como se muestra en la micrografía tomada (Figura 4). Las vesículas son micelas hinchadas o macromicelas, constituidas por capas alternas de agua y capas de surfactante conteniendo al aceite formando esferas dispersas en la fase en exceso (agua o aceite). ${ }^{12}$ Para el caso de la Figura 4, las vesículas corresponden a un sistema agua/surfactante/ aceite de composición 75:19:6.

De igual forma, se construyó el diagrama pseudoternario para observar el comportamiento de fase del sistema alcohol polietoxilado/agua/dodecano (Figura 5). Al igual que el diagrama anterior, se presentaron varias regiones monofásicas, bifásicas, multifásicas y vesículas, como se describen a continuación: 1) w/o: región de microemulsión agua-en-aceite. Más amplia que la obtenida con el decano, y se extiende a lo largo del eje surfactante/dodecano hasta una máxima composición de agua de $15 \%$ para la relación
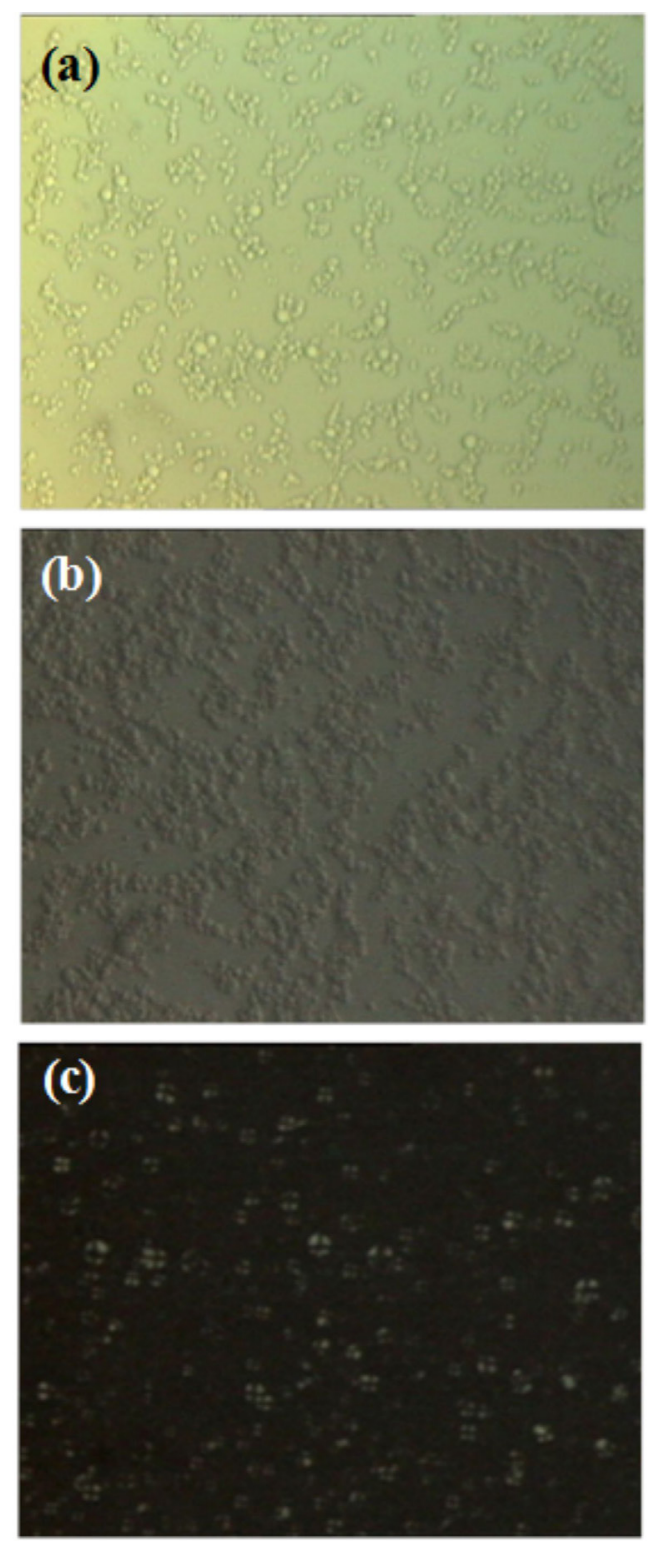

Figura 4. Micrografías (escala $20 \mu \mathrm{m}$ ) de la fase de (a) microemulsión tipo w/o para una composición agua/surfactante/decano 4:70:26, tomada con luz normal; (b) microemulsión tipo o/w para una composición agua/surfactantel decano 75:6:19 tomada con luz polarizada; (c) fase de vesículas o liposomas para el sistema surfactante/agua/decano

surfactante/dodecano igual a 45:40. Esta microemulsión está separada de una fase acuosa en exceso; 2) L $\alpha$ : región de cristales líquidos obtenida a una composición a lo largo del eje surfactante/agua entre 19:81 a 57:43, y hacia el centro del diagrama cuya composición máxima fue de $37 \%$ de dodecano para una relación surfactante/agua igual a 23:36; 3) ML $\alpha$ : región multifásica de microemulsiones w/o y o/w coexistiendo en equilibrio con una fase de cristales líquidos sin separarse, obtenida a composiciones con 52 a $90 \%$ de agua, y hacia el vértice del dodecano hasta un $37 \%$ para una relación surfactante/agua igual a 17:47;4) o/w: región de microemulsión aceite-en-agua, extendida en el eje dodecano/agua hasta un máximo de $13 \%$ de surfactante para una composición dodecano/agua 50:37, separada de una fase oleica en exceso; 5) $\mathrm{Fb}+\mathrm{L} \alpha$ : región de fase bicontinua compuesta por una fase de cristal líquido separada de una fase de microemulsión o/w y una fase de microemulsión w/o. Se encuentra en el centro del diagrama y se extiende con composiciones desde 13 a $87 \%$ de surfactante y hasta el vértice del dodecano. Esta 
fase fue birrefringente al movimiento; 6) V: región de vesículas o liposomas dispersas en medio acuoso. De mayor amplitud que la obtenida con el decano. Se extiende desde el vértice acuoso hasta un $55 \%$ de agua, y alcanza una concentración máxima de $7 \%$ de dodecano. Estos sistemas se separan en una fase de cristal líquido en equilibrio con una fase acuosa. La región de vesículas en fase oleica no se determinó con precisión.

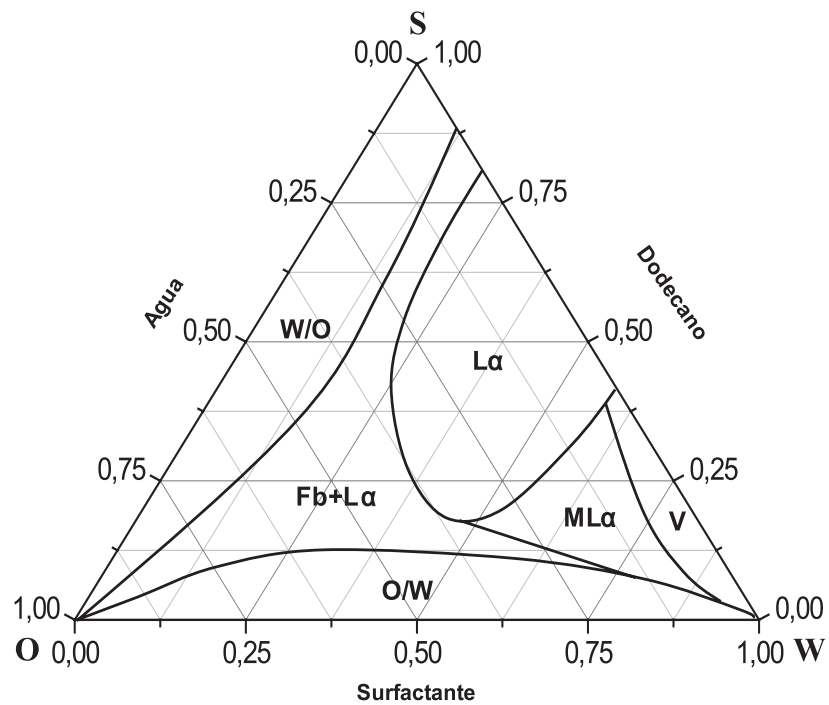

Figura 5. Diagrama ternario para el comportamiento de fases del sistema surfactantelagua/dodecano, a $25^{\circ} \mathrm{C}$

A través de la microscopía óptica se definieron las estructuras que formaron los sistemas surfactante/agua/dodecano. En la Figura 6 se presentan las micrografías de las fases de cristales líquidos para los sistemas con dodecano. Se encontraron cristales líquidos laminares con textura de líneas oleosas (Figura 6a) y de tipo hexagonal con estructuras de relieves (Figura 6b), para sistemas de composición surfactante/agua igual a 70:30 y 25:75, respectivamente. En la región multifásica $(\mathrm{ML} \alpha)$ se observaron cristales líquidos hexagonales en equilibrio con las fases de microemulsión (Figura 6c).

En el caso de los sistemas microemulsionados, se tomaron las micrografías de un sistema surfactante/agua/dodecano de composición igual a 70:5:25 (Figura 7a) correspondiente a una microemulsión w/o, y de composición 5:75:20 (Figura 7b) correspondiente a una microemulsión o/w, presentando ambas características similares en el tamaño de gotas como se puede notar en ambas micrografías. Se puede observar que estas microemulsiones no presentaron flóculos de gotas como se observó en los sistemas con decano (Figura 4).

En el caso de la fase de vesículas en fase acuosa (Figura 7c), éstas presentaron cruces de extinción de estructuras uniáxicas negativas características de este tipo de fases, que luego se separan en una fase de cristal líquido laminar. Las características generales de los diagramas estudiados con decano y dodecano coinciden con los de sistemas ternarios agua/éter alquílico polietilenglicol/aceite reportados en la literatura..$^{14,15}$ Debido a que el punto de turbidez de la solución agua/ surfactante es inferior a $25{ }^{\circ} \mathrm{C}$, los diagramas estudiados a esta temperatura no presentaron una región monofásica de micelas normales $\left(\mathrm{L}_{1}\right)$, en la región con alto contenido de agua.

Se puede observar que la región monofásica de cristales líquidos, L $\alpha$, tiene mayor extensión cuando se aumenta la longitud de la cadena hidrocarbonada del aceite, lo que concuerda con el hecho de que aumentan las fuerzas de atracción entre agregados micelares con el dodecano, incrementándose la rigidez de la película de surfactante que favorece la formación del cristal líquido. ${ }^{16-19}$ Como se esperaba, el sistema con mayor extensión de la región
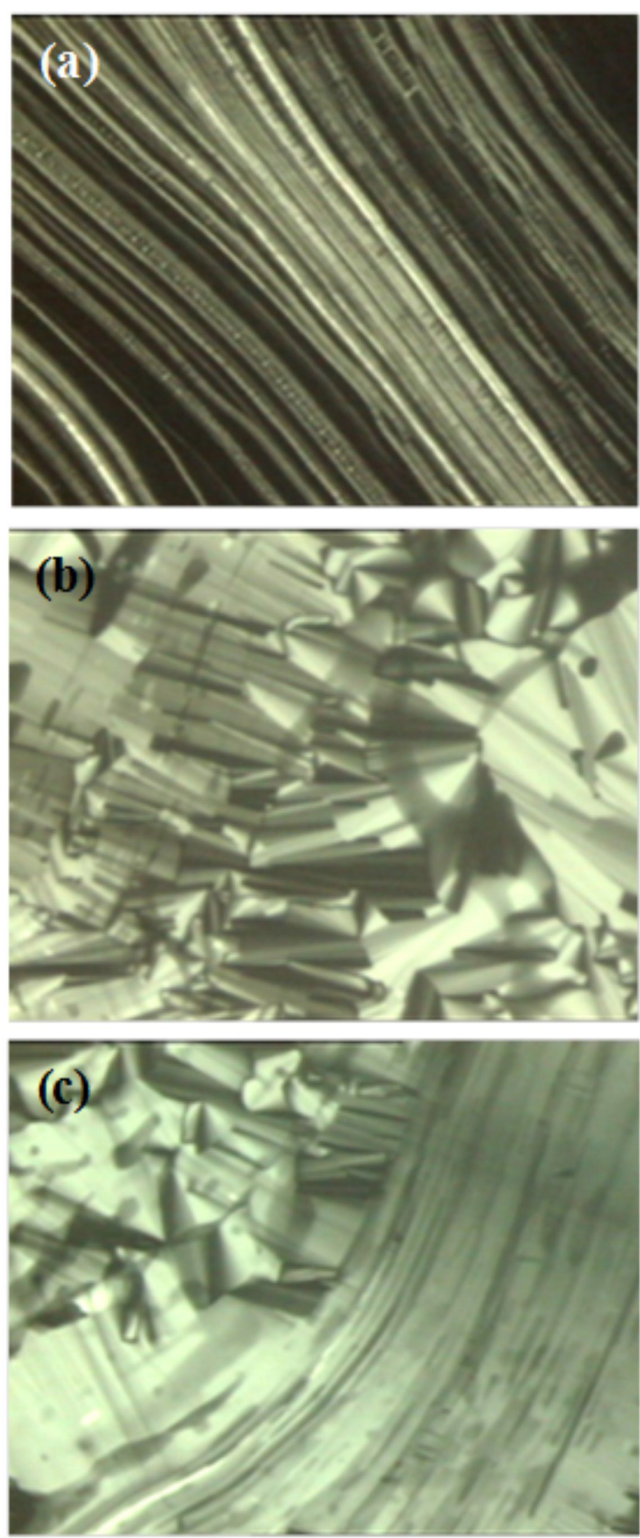

Figura 6. Micrografías (escala $20 \mu \mathrm{m}$ ) de la fase de cristal líquido: (a) tipo laminar, (b) tipo hexagonal; y (c) de la región multifásica, para el sistema surfactante/agua/dodecano

inmiscible o fases bicontinuas, correspondió al dodecano (de menor solubilidad y afinidad en agua). Este resultado coincide con los descritos en la literatura ${ }^{20-22}$ donde con los hidrocarburos de menor tamaño la interacción que promueve la miscibilidad del tensioactivo con las fases oleica y acuosa es menor comparada con las interacciones laterales entre las moléculas del tensioactivo, por ello la fase de cristal líquido tiende a formarse a concentraciones menores del surfactante.

Como se pudo observar en las micrografía presentadas, la fase de cristal líquido liotrópico está caracterizada por una geometría a nivel molecular a la cual, tanto laminares como hexagonales, les corresponde un valor denominado parámetro crítico de acomodamiento que como consecuencia del orden presente en el tipo de estructura son capaces de difractar los rayos X. ${ }^{3,6}$ De aquí que la técnica de difracción de rayos X en ángulo pequeño (SAXS) suministra información sobre la estructura de cada una de las fases de cristales líquidos. Debido a limitaciones en disponibilidad instrumental, en esta investigación no fue posible elucidar el tipo de mesofase por la técnica SAXS, sin embargo, la microscopía óptica con luz polarizada permitió determinar 

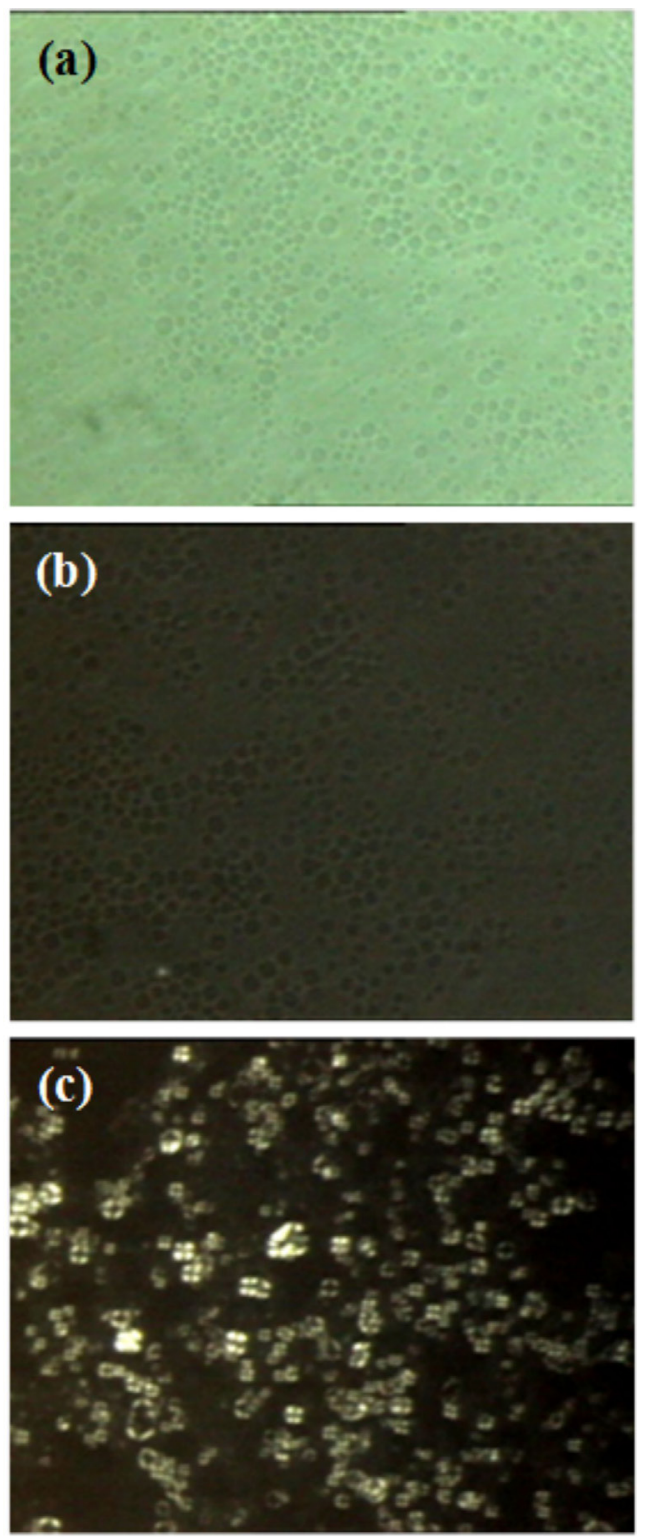

Figura 7. Micrografías (escala $20 \mu \mathrm{m}$ ) de la fase de microemulsión: (a) tipo w/o a una composición agua/surfactante/aceite 5:70:25, tomada con luz normal; (b) tipo o/w a una composición agua/surfactante/aceite 75:5:20 tomada con luz polarizada; (c) fase de vesículas o liposomas del sistema agua/surfactante/dodecano

con alta definición y adecuada precisión las texturas y estructuras de las fases de cristales líquidos.

\section{CONCLUSIONES}

El método que consistió en adición de agua a una mezcla surfactante/aceite constante, puede ser utilizado para estudiar el comportamiento de fase de sistemas surfactante polietoxilado/agua/aceite. Este método de dispersión facilita la obtención de cristales líquidos y sistemas microemulsionados. Tales sistemas poseen características físicas que pueden ser estudiadas por la técnica de microscopía óptica con luz polarizada, y por medio de la cual es posible conocer la estructura y tipo de fase obtenida. Los diagramas ternarios estudiados pueden ser modelos para el comportamiento de fase de otros sistemas con surfactante no iónicos para la formación de cristales líquidos, microemulsiones y, posiblemente, emulsiones submicrométricas o nanoemulsiones.

\section{AGRADECIMIENTOS}

Al Consejo de Desarrollo Científico y Humanístico de la Universidad del Zulia (CONDES-LUZ) por el financiamiento otorgado.

\section{REFERENCIAS BIBLIOGRÁFICAS}

1. Myers, D.; Surfaces, Interfaces, and Colloids: Principles and Applications, 2nd ed., Wiley: New York, 1999.

2. Holmberg, K.; Jonsson, B.; Kronberg, B.; Lindman, B.; Surfactants and Polymers in Aqueous Solution, 2nd ed., Wiley: England, 2002.

3. Márquez, N.; Bravo, B.; Chávez, G.; Ysambertt, F.; Salager, J. L. In Topics in Colloidal Aggregation and Interfacial Phenomena; GarciaSucre, M.; Lozsan, A.; Castellanos-Suarez, A.; Toro-Mendoza, J., eds.; Research Signpost: Kerala, 2012, chap. 6.

4. Raman, I. A.; Suhaimi, H.; Tiddy, G. J. T.; Adv. Colloid Interface Sci. 2003, 106, 109.

5. Solans, C.; Izquierdo, P.; Nolla, J.; Azemar, N.; Garcia-Celma, M. J; Curr. Opin. Colloid Interface Sci. 2005, 10, 102.

6. Pasquali, R. C.; Bregni, C.; Serrao, R.; Rev. Mex. Cienc. Farm. 2006, 37, 38.

7. Pasquali, R. C.; Bregni, C; Ars. Pharm. 2006, 47, 219.

8. Shinoda, K.; J. Colloid Interface Sci. 1967, 24, 4.

9. Kunieda, H.; Shinoda, K; J. Colloid Interface Sci. 1985, 107, 107.

10. Kunieda, H.; Nakamura, K.; Davis, H.T.; Evans, F.; Langmuir 1991, 7, 1915.

11. Strey, R.; Schomacker, R.; Roux, D.; Nallet, F.; Olsson, U.; J. Chem. Soc., Faraday Trans. 1990, 86, 2253.

12. Collings, P.J.; Hird, M.; Introduction to Liquid Crystal, Taylor \& Francis Group: London, 2004.

13. Collings, P.J.; Liquid Crystals, 2nd ed., Princeton University Press: Princeton, 2002.

14. Kahlweit, M.; Strey, R.; Busse, G; J. Phys. Chem. 1991, 95, 5344.

15. Kunieda, H.; Shinoda, K.; J. Dispersion Sci. Technol. 1982, 3, 233.

16. Bourrel, M.; Schechter, R.; Microemulsions and Related Systems, Marcel Dekker: New York, 1988.

17. Kunieda, H.; Ozawa, K.; Huang, K.; J. Phys. Chem. B 1998, 102, 831.

18. Olsson, U.; Shinoda, K.; Lindman, B.; J. Phys. Chem. 1986, 90, 4083.

19. Miller, C.; Raney, K.; Colloids Surf., A 1993, 74, 169.

20. Forgiarini, A.; Esquema, J.; González, C.; Solans, C.; Prog. Colloid Polym. Sci. 2000, 115, 36.

21. Forgiarini, A.; Esquema, J.; González, C.; Solans, C.; Langmuir 2001, 17, 2076.

22. Forgiarini, A.; Esquema, J.; González, C.; Solans, C.; Prog. Colloid Polym. Sci. 2001, 118, 184. 